\title{
Accurate and Efficient Model Energies for Exploring Intermolecular Interactions in Molecular \\ Crystals
}

Michael J.Turner, Simon Grabowsky, ${ }^{\S}$ Dylan Jayatilaka and Mark A. Spackman*

School of Chemistry \& Biochemistry, University of Western Australia, Crawley WA 6009, Australia

AUTHOR INFORMATION

Corresponding Author

*Email: mark.spackman@uwa.edu.au

Present Address

${ }^{\S}$ Universität Bremen, Fachbereich 2 - Biologie/Chemie, Leobener Str. NW2, 28359 Bremen, Germany 


\section{ABSTRACT}

The energy of interaction between molecules is commonly expressed in terms of four key components: electrostatic, polarization, dispersion and exchange-repulsion. Using monomer wavefunctions to obtain accurate estimates of electrostatic, polarization and repulsion energies, along with Grimme's dispersion corrections, a series of energy models are derived by fitting to dispersion-corrected DFT energies for a large number of molecular pairs extracted from organic and inorganic molecular crystals. The best performing model reproduces B3LYP-D2/6-31G(d,p) counterpoise-corrected energies with a mean absolute deviation (MAD) of just over $1 \mathrm{~kJ} \mathrm{~mol}^{-1}$, but in considerably less computation time. It also performs surprisingly well against benchmark $\mathrm{CCSD}(\mathrm{T}) / \mathrm{CBS}$ energies, with a MAD of $2.5 \mathrm{~kJ} \mathrm{~mol}^{-1}$ for a combined dataset including Hobza's X40, S22, A24 and S66 dimers. Two of these energy models - the most accurate, and the fastest are expected to find widespread application in investigations of molecular crystals.

\section{TOC GRAPHICS}

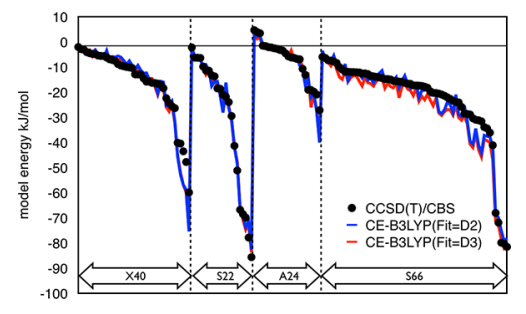

KEYWORDS dispersion-corrected DFT, molecular crystals, intermolecular interaction, dispersion, electrostatic, interaction energy 
In recent years our research has focused on the development of novel approaches, especially graphical ones based on Hirshfeld surface analysis, to investigate and rationalize intermolecular interactions in molecular crystals. ${ }^{1}$ Despite some attempts to extract quantitative information from the Hirshfeld surface, ${ }^{2-3}$ these approaches remain inherently qualitative. However, it has become clear to us $^{4}$ and others ${ }^{5-7}$ that mapping the molecular electrostatic potential on Hirshfeld surfaces can provide direct insight into intermolecular interactions in crystals. Close molecular contacts in the crystal can be rationalized and discussed in terms of the electrostatic complementarity of touching surface patches in adjacent molecules. ${ }^{4}$ The relative magnitudes of the electrostatic potentials mapped in this manner correlate with computed electrostatic energies for many different interactions. ${ }^{4}$

Over this same period investigations of molecular crystals in terms of pairwise interaction energies have become increasingly popular, in large part due to Gavezzotti's PIXEL approach, ${ }^{8-11}$ which has for some time provided a computationally tractable means of obtaining reliable intermolecular energies via a nonempirical electrostatic energy, along with semiempirical polarization, dispersion and repulsion contributions. Alternative quantum mechanical approaches, using various levels of theory and basis sets, have also been employed, especially in work by Shishkin and others, ${ }^{12-15}$ but these are necessarily more time-consuming for wide application to a large number and variety of molecular crystals.

In parallel with these developments, considerable attention has been very recently given to the computation of pairwise interaction and lattice energies for organic crystals ${ }^{16-22}$ and quite large supramolecular complexes ${ }^{23-24}$ using dispersion-corrected density functional theory. Here, we outline an approach to computing accurate model energies for use in crystal structure analysis. It has been inspired by the extensive work of Gavezzotti, whose PIXEL approach has become 
popular in applications to organic molecular crystals. ${ }^{6,25-27}$ Our objective is quite simple: to obtain an accurate and computationally efficient model for intermolecular interaction energies that can be applied as widely and efficiently as possible to organic and inorganic molecular crystals, and used in conjunction with our program CrystalExplorer. ${ }^{28}$

Our basic expression for the interaction energy follows that used by Gavezzotti and others, $E_{\text {tot }}=E_{\text {ele }}+E_{\text {pol }}+E_{\text {dis }}+E_{\text {rep }}$

where the terms have their usual meaning. This separation of contributions to the total interaction energy also follows many energy decomposition schemes, such as those of Kitaura \& Morokuma $^{29-30}$ or Hayes \& Stone, ${ }^{31}$ but here we construct the interaction energy from its individual components, rather than breaking down a much larger total energy for the interacting pair of molecules. This is philosophically closer to our desire to gain insight into packing of molecules in crystals using unperturbed electron densities and related properties. The terms in the interaction energy are specifically defined as follows:

$E_{\text {ele }}$ Classical electrostatic energy of interaction between unperturbed charge distributions of the monomers (from the antisymmetric product of the monomer spin orbitals);

$E_{p o l}$ Polarization energy as a sum over nuclei with terms of the kind $-\frac{1}{2} \alpha_{\text {mean }}|F|^{2}$, where the electric field at each nucleus is computed from the charge distribution of the other monomer, and $\alpha_{\text {mean }}$ are recommended isotropic atomic polarizabilities from Thakkar \& Lupinetti; ${ }^{32}$ 
$E_{\text {dis }}$ Grimme's D2 ${ }^{33}$ or $\mathrm{D} 3(\mathrm{BJ})^{34-35}$ dispersion correction, summed over all intermolecular atom pairs. Compared with D2 the D3(BJ) model employs a more elaborate method for choosing atomic dispersion coefficients and a different damping function.

$E_{\text {rep }}$ Exchange-repulsion energy, calculated between unperturbed charge distributions of the monomers (also from the antisymmetric product of the monomer spin orbitals, or spin natural orbitals with largest occupations for correlated wavefunctions). A more detailed exposition of this and the electrostatic term has been given recently by Su and $\mathrm{Li}^{36}$

The energies obtained in this manner are remarkably sensible, but they vary considerably from any meaningful quantum mechanical estimates (see the upper plots in Figs. 1 and 2, where they are labelled by the abbreviation CE for CrystalExplorer). To remedy this we introduce four scale factors, one for each term in the energy expression, and determine their optimum values by fitting to a large training set of quantum mechanical interaction energies based on dispersionand counterpoise-corrected B3LYP/6-31G(d,p) calculations. This training set contains 232 molecular pairs extracted from 37 organic and inorganic molecular crystal structures including atoms up to Xe. For these quantum mechanical energies two different dispersion models due to Grimme were explored: D2 ${ }^{33}$ and D3, ${ }^{34-35}$ the latter with Becke-Johnson damping. As the 6-31G basis is not defined for atoms beyond $\mathrm{Kr}$, calculations including those atoms used the DGDZVP basis, a practice shown by Gavezzotti to be an appropriate alternative. ${ }^{10}$ Further details are provided in the Supporting Information. Monomer electron densities used to compute $E_{\text {elec }}, E_{\text {pol }}$ and $E_{r e p}$ were obtained at three different levels of theory: HF/3-21G, MP2/6-31G(d,p) and B3LYP/6-31G(d,p). Figures 1 and 2 illustrate the effect of this simple scaling procedure, and the measures of fit and resulting scale factors are summarized in Table 1. 


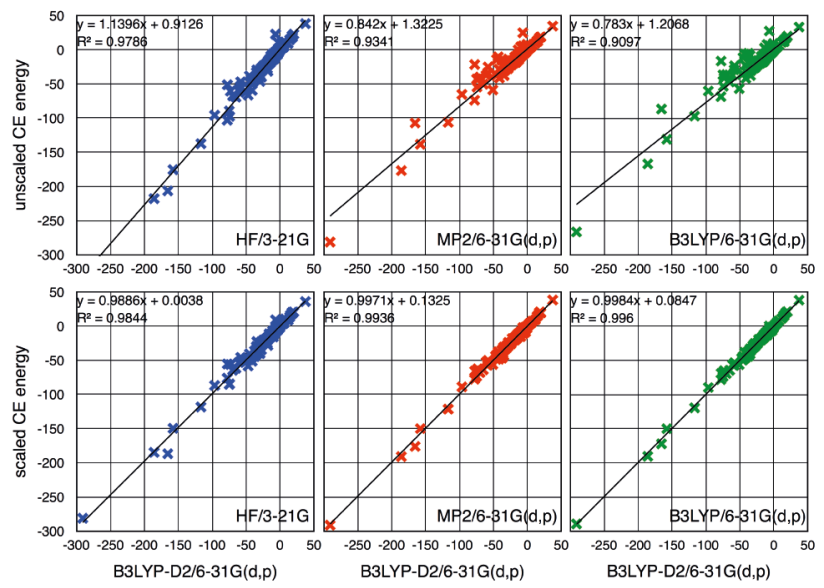

Figure 1. Scatter plots for 'unscaled' (top) and 'scaled' (bottom) model energies vs B3LYP$\mathrm{D} 2 / 6-31 \mathrm{G}(\mathrm{d}, \mathrm{p})$ counterpoise-corrected results. Plots and linear regression trendline data are provided for three different choices of monomer electron densities. All values are in $\mathrm{kJ} \mathrm{mol}^{-1}$, and note that the energies span a range of almost $350 \mathrm{~kJ} \mathrm{~mol}^{-1}$. Similar plots for fits to B3LYPD3(BJ)/6-31G(d,p) counterpoise-corrected energies are given in the Supporting Information.

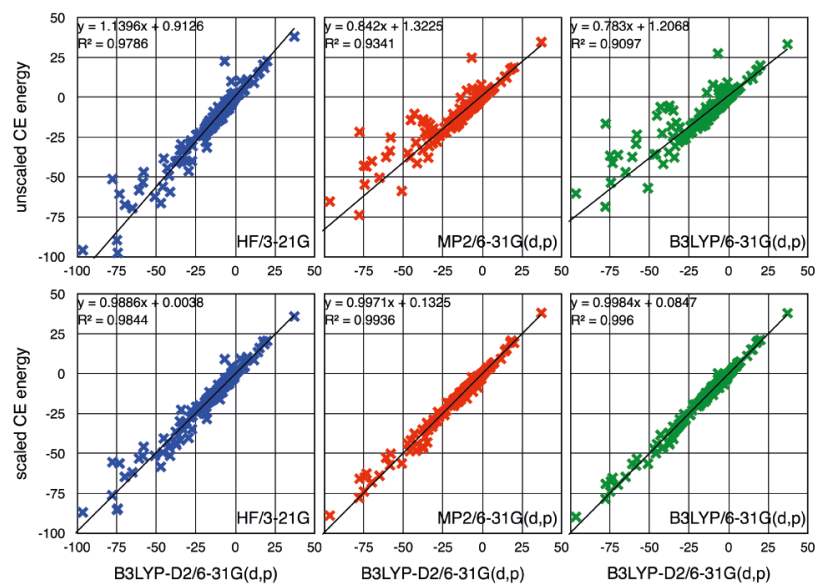

Figure 2. As for Figure 1, but limited to data between -100 and $+50 \mathrm{~kJ} \mathrm{~mol}^{-1}$. Similar plots for fits to B3LYP-D3(BJ)/6-31G(d,p) counterpoise-corrected energies are given in the Supporting Information. 
Table 1. MAD, MD, root-mean-square deviation (RMSD) and minimum/maximum deviations (in $\mathrm{kJ} \mathrm{mol}^{-1}$ ) for model energies, and optimum scale factors for individual energy components. Results are given for fits to both training sets of energies.

\begin{tabular}{|l|l|l|l|l|l|l|}
\hline & \multicolumn{3}{|l|}{ fits to B3LYP-D2/6-31G(d,p) energies } & \multicolumn{4}{l|}{ fits to B3LYP-D3(BJ)/6-31G(d,p) energies } \\
\hline $\begin{array}{l}\text { monomer } \\
\text { electron } \\
\text { density }\end{array}$ & \multicolumn{1}{|c|}{$\begin{array}{c}\text { HF } \\
3-21 G\end{array}$} & \multicolumn{1}{|c|}{$\begin{array}{c}\text { MP2 } \\
6-31 G(d, p)\end{array}$} & $\begin{array}{c}\text { B3LYP } \\
6-31 G(d, p)\end{array}$ & $\begin{array}{c}\text { HF } \\
3-21 G\end{array}$ & $\begin{array}{c}\text { MP2 } \\
6-31 G(d, p)\end{array}$ & $\begin{array}{c}\text { B3LYP } \\
6-31 G(d, p)\end{array}$ \\
\hline MAD & 2.1 & 1.4 & 1.2 & 2.1 & 1.3 & 1.2 \\
\hline MD & 0.2 & 0.2 & 0.1 & 0.2 & 0.2 & 0.1 \\
\hline RMSD & 4.0 & 2.5 & 2.0 & 3.9 & 2.5 & 2.0 \\
\hline $\begin{array}{l}\text { min, max } \\
\text { deviations }\end{array}$ & $-21.4,22.2$ & $-10.7,11.7$ & $-6.9,8.5$ & $-22.5,19.8$ & $-11.2,10.2$ & $-8.1,9.2$ \\
\hline$k_{\text {ele }}$ & 0.882 & 1.027 & 1.063 & 0.884 & 1.039 & 1.060 \\
\hline$k_{\text {pol }}$ & 0.593 & 0.722 & 0.756 & 0.540 & 0.656 & 0.729 \\
\hline$k_{\text {dis }}$ & 0.852 & 0.842 & 0.843 & 0.967 & 0.970 & 0.963 \\
\hline$k_{\text {rep }}$ & 0.681 & 0.605 & 0.595 & 0.637 & 0.586 & 0.573 \\
\hline
\end{tabular}

This simple scaling approach is clearly extremely successful, as seen by the quality of fits to the training sets of energies (Figures 1 and 2), but also the measures of fit (Table 1). In all cases the mean deviation (MD) is effectively zero, and the mean absolute deviation (MAD) is between 1.2 and $2.1 \mathrm{~kJ} \mathrm{~mol}^{-1}$, for a set of reference energies spanning a range of nearly $350 \mathrm{~kJ} \mathrm{~mol}^{-1}$ (and for which the mean absolute reference energy is $\left.18.4 \mathrm{~kJ} \mathrm{~mol}^{-1}\right)$. Perhaps unsurprisingly, the best fit is obtained using B3LYP/6-31G $(\mathrm{d}, \mathrm{p})$ monomer electron densities, but MP2/6-31G(d,p) densities result in only a slightly worse fit and, quite surprisingly, even $\mathrm{HF} / 3-21 \mathrm{G}$ electron 


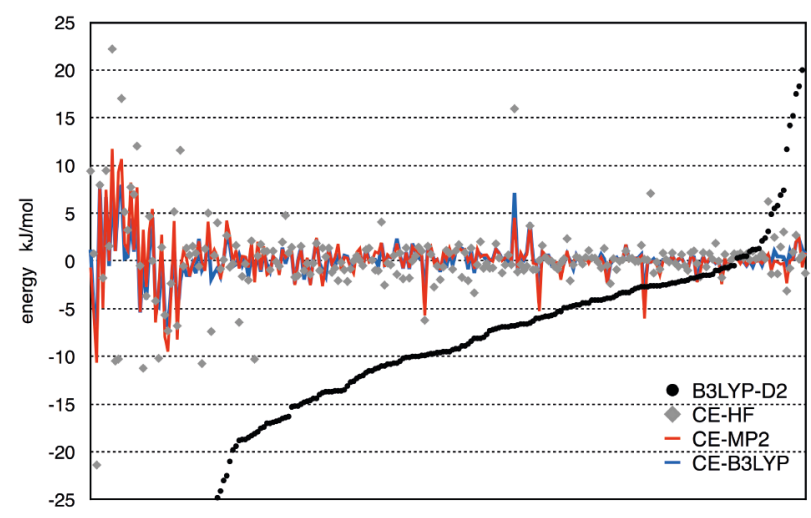

Figure 3. Deviation of model energies from training set values for Fit=D2. The data are ordered from left to right by decreasing magnitude of the reference interaction energy, values for which are shown as black circles where they fit within the range of the plot. Data on the far left refer to large negative interaction energies, while those on the far right refer to positive interaction energies.

densities are capable of providing meaningful estimates of interaction energies, albeit with much larger deviations from the training data.

The sets of scale factors in Table 1 can be considered to specify six different model energies, and from here onwards it is economical to refer to them by the abbreviations CE-HF(Fit=D2), CE-MP2(Fit=D3) etc., where CE is a reference to our program CrystalExplorer, ${ }^{28} \mathrm{HF}, \mathrm{MP} 2$ and B3LYP refer to the theory level used to obtain monomer electron densities (the basis sets are implicitly those given in Table 1, or, as previously discussed, DGDZVP rather than 6-31G(d,p) for atoms larger than $\mathrm{Kr}$ ), and D2 and D3 refer to the specific dispersion correction employed in the training set of energies (where B3LYP-D2/6-31G(d,p) and B3LYP-D3(BJ)/6-31G(d,p) are implied). In Figure 3 we depict graphically the deviation of CE-HF, CE-MP2 and CE-B3LYP energies (all Fit=D2) from those for the B3LYP-D2/6-31G(d,p) training set. With few 
exceptions, the largest deviations are found for molecular pairs with the most negative interaction energies.

The scale factors also reveal some interesting trends, notably the constancy of $k_{d i s}$, irrespective of the choice of electron density, but differences are observed between D2 and D3(BJ) dispersion models, as expected. Although the statistics in Table 1 suggest the preferred model energies are those based on B3LYP/6-31G(d,p) monomer electron densities, there is little difference between fits to D2 or D3(BJ) dispersion corrected quantum mechanical results (i.e. either CE-B3LYP(Fit=D2) or CE-B3LYP(Fit=D3)).

Table 2. Statistical results for comparisons between benchmark CCSD(T)/CBS energies and CEB3LYP(Fit=D2) or CE-B3LYP(Fit=D3) model energies. Values are given in $\mathrm{kcal}^{\mathrm{mol}}{ }^{-1}$ for ease of comparison with literature results.

\begin{tabular}{|l|c|c|c|c|c|c|c|}
\hline & \multicolumn{3}{|c|}{ CE-B3LYP(Fit=D2) } & \multicolumn{3}{c|}{ CE-B3LYP(Fit=D3) } \\
\hline & MAD & MD & RMSD & & MAD & MD & RMSD \\
\hline X40 & 0.70 & 0.18 & 1.12 & & 0.62 & 0.30 & 1.07 \\
\hline S22 & 0.54 & 0.02 & 0.73 & & 0.44 & 0.14 & 0.61 \\
\hline A24 & 0.42 & 0.28 & 0.81 & & 0.47 & 0.27 & 0.86 \\
\hline S66 & 0.61 & 0.33 & 0.88 & & 0.88 & 0.85 & 1.12 \\
\hline all 152 pairs & 0.60 & 0.24 & 0.92 & & 0.68 & 0.51 & 1.01 \\
\hline
\end{tabular}

To further examine the potential capability of these two model energies we have computed interaction energies for pairs of molecules in several standard benchmark datasets for intermolecular interactions: $\mathrm{X} 40,{ }^{37} \mathrm{~S} 22,{ }^{38} \mathrm{~A} 24^{39}$ and $\mathrm{S} 66 .{ }^{40}$ For this purpose we have used the geometries provided in the Benchmark Energy \& Geometry Database ${ }^{41}$ and compare our model results with $\mathrm{CCSD}(\mathrm{T}) / \mathrm{CBS}$ energies tabulated there. Table 2 summarizes the MAD, MD and 
RMSD, and Figure 3 compares graphically the trends obtained with CE-B3LYP(Fit=D2) and CE-B3LYP(Fit=D3) model energies against the CCSD(T)/CBS benchmark values for each of the four datasets.

Uniformly positive MD values in Table 2 indicate that the present CE-B3LYP(Fit=D3) model energies yield systematic overestimates of benchmark binding energies, and this is confirmed by the trends displayed in Figure 4, where the CE-B3LYP(Fit=D3) results lie below both benchmark and CE-B3LYP(Fit=D2) for many molecular pairs in the S66 dataset. Combined with statistics for the complete set of energies in Table 2, we conclude that the CE-B3LYP(Fit=D2) model is to be preferred over CE-B3LYP(Fit=D3) for our purposes, and our subsequent discussion focuses only on this model.

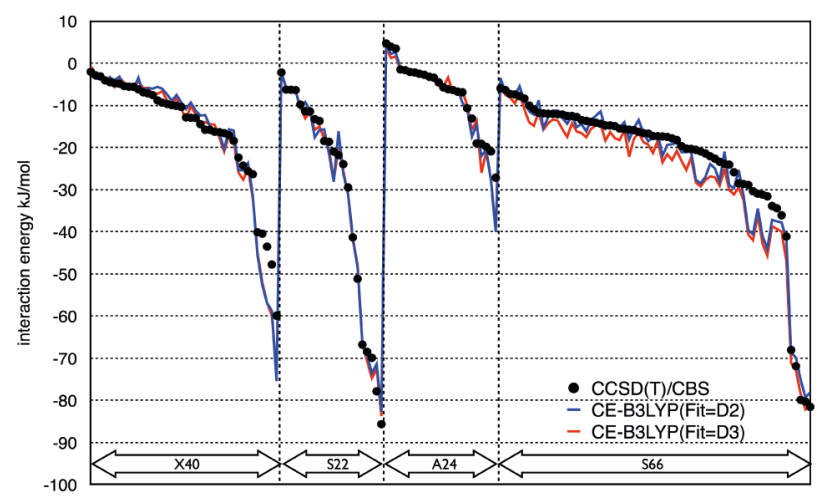

Figure 4. Comparison between model energies and $\operatorname{CCSD}(\mathrm{T}) / \mathrm{CBS}$ benchmark values. For each benchmark set the data have been sorted by increasing magnitude of the interaction energy.

At this point it is useful to compare the capability of this preferred energy model against $\operatorname{CCSD}(\mathrm{T})$ benchmark values with the performance of a range of DFT-D and wavefunction-based methods in the recent literature. For the S66 set Goerigk et al. ${ }^{17}$ have reported MAD values between 0.19 and $0.40 \mathrm{kcal} \mathrm{mol}^{-1}$ for a wide range of DFT-D methods using a QZVP basis, while 
values as large as 0.45 and $0.74 \mathrm{kcal} \mathrm{mol}^{-1}$ have been reported for MP2/CBS and SCSMP2/CBS, respectively. ${ }^{40}$ The present CE-B3LYP(Fit=D2) MAD of $0.61 \mathrm{kcal} \mathrm{mol}^{-1}$ compares $^{-1}$ favorably. For the S22 set Carter and Rohl $^{18}$ have reported MAD values between 0.55 and 1.60 $\mathrm{kcal} \mathrm{mol}^{-1}$ for a range of basis sets and GGA functionals with vdW-DF dispersion. The present CE-B3LYP(Fit=D2) result of $0.54 \mathrm{kcal} \mathrm{mol}^{-1}$ again compares favorably, and even more so against results for other methods summarized in Table 3 of that work.

The performance of the PM6 and PM6-DH2 semiempirical schemes against S22, S66 and X40 sets has been reported recently by Sure and Grimme, along with results obtained with their proposed small basis set corrected Hartree-Fock method, HF-3c. ${ }^{42}$ For these methods MAD values reported are (in $\mathrm{kcal} \mathrm{mol}^{-1}$ for HF-3c, PM6 and PM6-DH2, respectively): X40; 1.44, 1.73 and 1.46; S22: 0.55, 3.39 and 0.39; S66: 0.38, 2.68 and 0.65. For comparison, MAD values obtained with the CE-B3LYP(Fit=D2) energy model are $0.70,0.54$ and $0.61 \mathrm{kcal} \mathrm{mol}^{-1}$, for X40, S22 and S66, respectively.

As a final test of the capability of our model energies, we have applied these methods to nine of the twelve large supramolecular complexes taken from the $\mathrm{S} 12 \mathrm{~L}^{23-24}$ set (we have omitted the cationic complexes $6 \mathrm{a}, 6 \mathrm{~b}$ and $7 \mathrm{a}$ ). For this purpose we test the performance of CEB3LYP(Fit=D2), as well as that of the less accurate but much faster CE-HF(Fit=D2), which is based on HF/3-21G monomer electron densities. Table 3 lists the energies obtained, and it emerges that both models overestimate the binding energies for all of these complexes, but the MAD from the empirical reference values is still under $5 \mathrm{kcal} \mathrm{mol}^{-1}$ for both models. Of particular interest is the fact that $\mathrm{CE}-\mathrm{HF}(\mathrm{Fit}=\mathrm{D} 2)$ energies are very close to those obtained from the CE-B3LYP(Fit=D2) model, but in considerably less time. It is also important to note that the energy differences for pairs of complexes with the same host molecule are faithfully reproduced 
in all cases by both of these models. These results can also be put into perspective by reference to some of the MAD values given by Risthaus and Grimme for a large number of DFT, MP2based and semiempirical methods for all of the S12L set. ${ }^{24}$ For example, they list a series of "workable" DFT methods, for which MAD values (in $\mathrm{kcal} \mathrm{mol}^{-1}$ ) range between 1.6 (PBED2/TZVP) and 5.4 (PBE-XDM/mixed TZ), and "best estimate" methods, for which the range of MAD values is 2.1 (PBE-NL/QZVP) to 4.1 (M06-L/QZVP). Once more we see that the two model energies tested here compare favorably.

Table 3. CE-B3LYP(Fit=D2) and CE-HF(Fit=D2) model energies compared with empirical reference energies ${ }^{24}$ for a subset of the S12L test set. In line with other tabulations from the literature, the present model energies include a three-body dispersion correction, ${ }^{23}$ and values are given in $\mathrm{kcal} \mathrm{mol}^{-1}$ for ease of comparison with literature results ${ }^{a}$

\begin{tabular}{|l|l|c|c|c|}
\hline complex & & CE-B3LYP(Fit=D2) & CE-HF(Fit=D2) & $\begin{array}{l}\text { empirical } \\
\text { reference }\end{array}$ \\
\hline 2a & TCNQ@tweezer & -31.8 & -33.5 & -29.9 \\
\hline 2b & DCB@tweezer & -21.9 & -21.7 & -20.5 \\
\hline 3a & 3c@pincer & -33.3 & -32.8 & -24.3 \\
\hline 3b & 3d@pincer & -28.0 & -29.2 & -20.4 \\
\hline 4a & C60@catcher & -29.4 & -30.4 & -27.5 \\
\hline 4b & C70@catcher & -30.5 & -31.0 & -28.7 \\
\hline 5a & GLH@mcycle & -41.5 & -42.2 & -34.8 \\
\hline 5b & BQ@mcycle & -28.1 & -28.7 & -23.1 \\
\hline 7b & ADOH@CB7 & -25.5 & -25.6 & -22.6 \\
\hline & & 4.2 & 4.8 & \\
\hline
\end{tabular}

${ }^{a}$ There appears to be a typographical error in Table 1 of reference 24 ; the empirical reference energy for complex $5 \mathrm{~b}$ is actually -23.1 and not $-21.3 \mathrm{kcal} \mathrm{mol}^{-1}$ as reported. 
The performance of these relatively simple models for interaction energies is remarkable, given that the four scale factors are the only "variables" in each model, with no special terms introduced to cater for hydrogen bonding, which is sometimes a feature of similar methods. The models have been derived from dispersion-corrected DFT energies for molecular pairs found in a very wide range of molecular crystals, inorganic as well as organic, and including atoms up to iodine and xenon. The success of this approach is perhaps in large part due to the considerable amount of quantum mechanics built into three of the energy terms, and the fact that Grimme's dispersion corrections have been optimised and soundly calibrated in many studies (although they were not calibrated against our training set). What is perhaps most significant is that the computation time associated with these models is much less than that associated with its corresponding ab initio training set. We expect that they will be of great benefit in quantitatively exploring, and thus developing a sound intuition for, interaction energies in molecular crystals, a goal that has long been advocated as necessary for crystal engineering. ${ }^{43}$

We find it interesting that the present $\mathrm{CE}$ model energies cannot really be labelled semiempirical, nor can they be called DFT or ab initio, or even a version of intermolecular perturbation theory. They perhaps represent a new hybrid method, with some similarity to the approach used by Li et al. who investigated linear scaling of SAPT0 energy terms. ${ }^{44}$ The present $E_{e l e}$ and $E_{\text {rep }}$ energies are equivalent, or very close, to the first terms in the SAPT expansion for electrostatic and exchange energies (for example, see reference 45), but in our case polarization and dispersion energies are not wavefunction-based. There are many improvements that could be made to the models, but we expect that increasing the size of the basis set is not likely to be helpful; it will only lead to longer computation times. Apart from fitting to a "better" set of training energies, the most attractive refinement is likely to involve replacing the isotropic 
polarizabilities used to determine $E_{\text {pol }}$ with distributed polarizabilities derived from the monomer wavefunctions.

We anticipate that the models presented here may find widespread application. We are currently exploring their use in a novel graphical representation of pairwise interaction energies in molecular crystals (unpublished results), which provides ready insight into some of the physical properties of crystals, such as cleavage and various mechanical properties. Future work involving these models will focus on their use in estimating lattice energies - and comparison with experimental values, as well as those from recent computational methods - as well as the extension to charged species such as organic salts.

\section{COMPUTATIONAL METHODS}

Energies for the training sets used the implementation of Grimme's D2 and D3 dispersion corrections (EmpiricalDispersion=GD2 and EmpiricalDispersion=GD3BJ, respectively) and counterpoise correction for basis set superposition error in Gaussian09. ${ }^{46}$ For the D2 corrections the overall scale factor $s_{6}$ was set to 1.0 instead of the default value of 1.05 , as recommended recently. ${ }^{16}$ All other computations were performed with CrystalExplorer $3.2,{ }^{28}$ using a local implementation of $\mathrm{D} 2$ dispersion, and $d f t d 3^{34-35}$ for the $\mathrm{D} 3(\mathrm{BJ})$ dispersion corrections. The preferred energy models (Fit=D2) have been incorporated into CrystalExplorer in such a way that monomer wavefunctions need to be computed only once for any pre-selected cluster surrounding a central molecule. Density matrices of neighboring molecules are generated by the various space group symmetry operations, and computation of interaction energies of a molecule with those in its first coordination sphere is completely automated. 


\section{ASSOCIATED CONTENT}

Supporting Information. Details of crystal structures and procedures used to determine interaction energies of molecular pairs for training sets. Scatter plots for fits to B3LYP$\mathrm{D} 3(\mathrm{BJ}) / 6-31 \mathrm{G}(\mathrm{d}, \mathrm{p})$ counterpoise-corrected energies. This material is available free of charge via the Internet at http://pubs.acs.org.

\section{AUTHOR INFORMATION}

\section{Corresponding Author}

*Email: mark.spackman@uwa.edu.au

\section{Notes}

The authors declare no competing financial interests.

\section{ACKNOWLEDGMENT}

The authors are grateful to Dr Amir Karton for helpful discussions on many aspects of this work, which has been supported by the Australian Research Council (DP130103304) and the Danish National Research Foundation (Center for Materials Crystallography, DNRF-93).

\section{REFERENCES}

(1) Spackman, M. A.; Jayatilaka, D. Hirshfeld Surface Analysis. CrystEngComm 2009, 11, 19-32.

(2) McKinnon, J. J.; Jayatilaka, D.; Spackman, M. A. Towards Quantitative Analysis of Intermolecular Interactions with Hirshfeld Surfaces. Chem. Commun. 2007, 3814-3816. 
(3) Grabowsky, S.; Dean, P. M.; Skelton, B. W.; Sobolev, A. N.; Spackman, M. A.; White, A. H. Crystal Packing in the 2-R,4-Oxo-1,3-a/b-naphthodioxanes - Hirshfeld Surface Analysis and Melting Point Correlation. CrystEngComm 2012, 14, 1083-1093.

(4) Spackman, M. A.; McKinnon, J. J.; Jayatilaka, D. Electrostatic Potentials Mapped on Hirshfeld Surfaces Provide Direct Insight into Intermolecular Interactions in Crystals. CrystEngComm 2008, 10, 377-388.

(5) Aldridge, S.; Downs, A. J.; Tang, C. Y.; Parsons, S.; Clarke, M. C.; Johnstone, R. D. L.; Robertson, H. E.; Rankin, D. W. H.; Wann, D. A. Structures and Aggregation of the Methylamine-borane Molecules $\mathrm{Me}_{n} \mathrm{H}_{3-\mathrm{n}} \cdot \mathrm{BH}_{3}(\mathrm{n}=1,3)$, Studied by X-ray Diffraction, Gas-phase Electron Diffraction, and Quantum Chemical Calculations. J. Am. Chem. Soc. 2009, 131, 22312243.

(6) Jarzembska, K. N.; Hoser, A. A.; Kaminski, R.; Madsen, A. O.; Durka, K.; Wozniak, K. Combined Experimental and Computational Studies of Pyrazinamide and Nicotinamide in the Context of Crystal Engineering and Thermodynamics. Cryst. Growth Des. 2014, 14, 3453-3465.

(7) Panini, P.; Chopra, D. Experimental and Theoretical Characterization of Short H-Bonds with Organic Fluorine in Molecular Crystals. Cryst. Growth Des. 2014, 14, 3155-3168.

(8) Gavezzotti, A. Calculation of Intermolecular Interaction Energies by Direct Numerical Integration over Electron Densities. 1. Electrostatic and Polarization Energies in Molecular Crystals. J. Phys. Chem. B 2002, 106, 4145-4154. 
(9) Gavezzotti, A. Calculation of Intermolecular Interaction Energies by Direct Numerical Integration over Electron Densities. 2. An Improved Polarization Model and the Evaluation of Dispersion and Repulsion Energies. J. Phys. Chem. B 2003, 107, 2344-2353.

(10) Gavezzotti, A. Non-conventional Bonding between Organic Molecules. The 'Halogen Bond' in Crystalline Systems. Mol. Phys. 2008, 106, 1473-1485.

(11) Maschio, L.; Civalleri, B.; Ugliengo, P.; Gavezzotti, A. Intermolecular Interaction Energies in Molecular Crystals: Comparison and Agreement of Localized Møller-Plesset 2, Dispersion-corrected Density Functional, and Classical Empirical Two-body Calculations. J. Phys. Chem. A 2011, 115, 11179-11186.

(12) Shishkin, O. V.; Dyakonenko, V. V.; Maleev, A. V. Supramolecular Architecture of Crystals of Fused Hydrocarbons Based on Topology of Intermolecular Interactions. CrystEngComm 2012, 14, 1795-1804.

(13) Shishkin, O. V.; Medvediev, V. V.; Zubatyuk, R. I.; Shyshkina, O. O.; Kovalenko, N. V.; Volovenko, J. M. Role of Different Molecular Fragments in Formation of the Supramolecular Architecture of the Crystal of 1,1-Dioxo-tetrahydro-1 $\lambda^{6}$-thiopyran-3-one. CrystEngComm 2012, $14,8698-8707$.

(14) Shishkin, O. V.; Medvediev, V. V.; Zubatyuk, R. I. Supramolecular Architecture of Molecular Crystals Possessing Shearing Mechanical Properties: Columns versus Layers. CrystEngComm 2013, 15, 160-167.

(15) Shishkin, O. V.; Zubatyuk, R. I.; Shishkina, S. V.; Dyakonenko, V. V.; Medviediev, V. V. Role of Supramolecular Synthons in the Formation of the Supramolecular Architecture of 
Molecular Crystals Revisited from an Energetic Viewpoint. Phys. Chem. Chem. Phys. 2014, 16, 6773-6786.

(16) Civalleri, B.; Zicovich-Wilson, C. M.; Valenzano, L.; Ugliengo, P. B3LYP Augmented with an Empirical Dispersion Term (B3LYP-D*) as Applied to Molecular Crystals. CrystEngComm 2008, 10, 405-410.

(17) Goerigk, L.; Kruse, H.; Grimme, S. Benchmarking Density Functional Methods against the S66 and S66x8 Datasets for Non-Covalent Interactions. ChemPhysChem 2011, 12, 34213433.

(18) Carter, D. J.; Rohl, A. L. Noncovalent Interactions in SIESTA Using the vdW-DF Functional: S22 Benchmark and Macrocyclic Structures. J. Chem. Theory Comput. 2012, 8, 281289.

(19) Otero de la Roza, A.; Johnson, E. J. A Benchmark for Non-Covalent Interactions in Solids. J. Chem. Phys. 2012, 137, 054103.

(20) Moellmann, J.; Grimme, S. DFT-D3 Study of Some Molecular Crystals. J. Phys. Chem. C 2014, 118, 7615-7621.

(21) Carter, D. J.; Rohl, A. L. Benchmarking Calculated Lattice Parameters and Energies of Molecular Crystals Using van der Waals Density Functionals. J. Chem. Theory Comput. 2014, $10,3423-3437$.

(22) Brandenburg, J. G.; Grimme, S. Accurate Modeling of Organic Molecular Crystals by Dispersion-Corrected Density Functional Tight Binding (DFTB). J. Phys. Chem. Lett. 2014, 5, 1785-1789. 
(23) Grimme, S. Supramolecular Binding Thermodynamics by Dispersion-Corrected Density Functional Theory. Chem. Eur. J. 2012, 18, 9955-9964.

(24) Risthaus, T.; Grimme, S. Benchmarking of London Dispersion-Accounting Density Functional Theory Methods on Very Large Molecular Complexes. J. Chem. Theory Comput. 2013, $9,1580-1591$.

(25) Fabbiani, F. P. A.; Buth, G.; Levendis, D. C.; Cruz-Cabez, A. J. Pharmaceutical Hydrates under Ambient Conditions from High-Pressure Seeds: A Case Study of GABA Monohydrate. Chem. Commun. 2014, 50, 1817-1819.

(26) Johnston, B. F.; Marshall, W. G.; Parsons, S.; Urquhart, A. J.; Oswald, I. D. H. Investigation of Acrylic Acid at High Pressure Using Neutron Diffraction. J. Phys. Chem. B 2014, 118, 4044-4051.

(27) Maloney, A. G. P.; Wood, P. A.; Parsons, S. Competition between Hydrogen Bonding and Dispersion Interactions in the Crystal Structures of the Primary Amines. CrystEngComm 2014, 16, 3867-3882.

(28) Wolff, S. K.; Grimwood, D. J.; McKinnon, J. J.; Turner, M. J.; Jayatilaka, D.; Spackman, M. A. CrystalExplorer 3.2, University of Western Australia. Perth, 2014.

(29) Kitaura, K.; Morokuma, K. New Energy Decomposition Scheme for Molecular Interactions within Hartree-Fock Approximation. Int. J. Quantum Chem. 1976, 10, 325-340.

(30) Morokuma, K.; Kitaura, K. Energy Decomposition Analysis of Molecular Interactions. In Chemical Applications of Atomic and Molecular Electrostatic Potentials, Politzer, P.; Truhlar, D. G., Eds. Plenum: New York, 1981; pp 215-242. 
(31) Hayes, I. C.; Stone, A. J. An Intermolecular Perturbation-Theory for the Region of Moderate Overlap. Mol. Phys. 1984, 53, 83-105.

(32) Thakkar, A. J.; Lupinetti, C. Atomic Polarizabilities and Hyperpolarizabilities: A Critical Compilation. In Atoms, Molecules and Clusters in Electric Fields, Maroulis, G., Ed. World Scientific: 2006; pp 505-529.

(33) Grimme, S. Semiempirical GGA-Type Density Functional Constructed with a LongRange Dispersion Correction. J. Comput. Chem. 2006, 27, 1787-1799.

(34) Grimme, S.; Antony, J.; Ehrlich, S.; Krieg, H. A Consistent and Accurate Ab Initio Parametrization of Density Functional Dispersion Correction (DFT-D) for the 94 Elements H-Pu. J. Chem. Phys. 2010, 132, 154104.

(35) Grimme, S.; Ehrlich, S.; Goerigk, L. Effect of the Damping Function in Dispersion Corrected Density Functional Theory. J. Comput. Chem. 2011, 32, 1456-1465.

(36) Su, P.; Li, H. Energy Decomposition Analysis of Covalent Bonds and Intermolecular Interactions. J. Chem. Phys. 2009, 131, 014102.

(37) Rezac, J.; Riley, K. E.; Hobza, P. Benchmark Calculations of Noncovalent Interactions of Halogenated Molecules. J. Chem. Theory Comput. 2012, 8, 4285-4292.

(38) Jurecka, P.; Sponer, J.; Cerny, J.; Hobza, P. Benchmark Database of Accurate (MP2 and $\operatorname{CCSD}(\mathrm{T})$ Complete Basis Set Limit) Interaction Energies of Small Model Complexes, DNA Base Pairs, and Amino Acid Pairs. Phys. Chem. Chem. Phys. 2006, 8, 1985-1993. 
(39) Rezac, J.; Hobza, P. Describing Noncovalent Interactions beyond the Common Approximations: How Accurate Is the "Gold Standard," $\operatorname{CCSD}(\mathrm{T})$ at the Complete Basis Set Limit? J. Chem. Theory Comput. 2013, 9, 2151-2155.

(40) Rezac, J.; Riley, K. E.; Hobza, P. S66: A Well-balanced Database of Benchmark Interaction Energies Relevant to Biomolecular Structures. J. Chem. Theory Comput. 2011, 7, 2427-2438.

(41) Rezac, J.; Jurecka, P.; Riley, K. E.; Cerny, J.; Valdes, H.; Pluhackova, K.; Berka, K.;

Rezac, T.; Pitonak, M.; Vondrasek, J. et al. Quantum Chemical Benchmark Energy and Geometry Database for Molecular Clusters and Complex Molecular Systems (http://www.begdb.com): A Users Manual and Examples. Collect. Czech. Chem. Commun. 2008, $73,1261-1270$.

(42) Sure, R.; Grimme, S. Corrected Small Basis Set Hartree-Fock Method for Large Systems. J. Comput. Chem. 2013, 34, 1672-1685.

(43) Dunitz, J. D.; Gavezzotti, A. Supramolecular Synthons: Validation and Ranking of Intermolecular Interaction Energies. Cryst. Growth Des. 2012, 12, 5873-5877.

(44) Li, A.; Muddana, H. S.; Gilson, M. K. Quantum Mechanical Calculation of Noncovalent Interactions: A Large-Scale Evaluation of PMx, DFT, and SAPT Approaches. J. Chem. Theory Comput. 2014, 10, 1563-1575.

(45) Hohenstein, E. G.; Sherrill, C. D. Wavefunction methods for noncovalent interactions. WIREs Comput. Mol. Sci. 2012, 2, 304-326. 
(46) Frisch, M. J.; Trucks, G. W.; Schlegel, H. B.; Scuseria, G. E.; Robb, M. A.; Cheeseman,

J. R.; Scalmani, G.; Barone, V.; Mennucci, B.; Petersson, G. A. et al. Gaussian 09, Revision D.01, Gaussian, Inc.: Wallingford, CT, USA, 2009. 